\title{
Governança de água e planejamento urbano: aproveitamento de água de chuva para construção de cidades mais resilientes
}

\author{
Water governance and urban planning: harnessing \\ rainwater to build more resilient cities
}

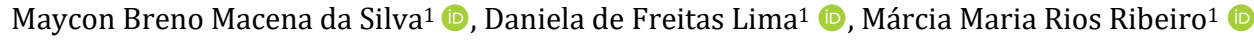

${ }^{1}$ Universidade Federal de Campina Grande - UFCG, Campina Grande, PB, Brasil. E-mails: sbrenomacena@gmail.com, danielafreitas1218@gmail.com,mm-ribeiro@uol.com.br

Como citar: Silva, M. B. M., Lima, D. F., \& Ribeiro, M. M. R. (2021). Governança de água e planejamento urbano: aproveitamento de água de chuva para construção de cidades mais resilientes. Revista de Gestão de Água da América Latina, 18, e18.

https://doi.org/10.21168/rega.v18e18

\begin{abstract}
RESUMO: As mudanças climáticas e o aumento da população urbana são fortes estressores para os recursos hídricos, fazendo-se necessário dialogar sobre a construção de sistemas urbanos resilientes e sensíveis à água. Desse modo, o aproveitamento de águas pluviais se apresenta como importante alternativa de acesso à água e de redução das pressões nos mananciais. Esta pesquisa objetivou identificar a potencialidade da captação da água de chuva em treze cidades do Estado da Paraíba por meio de simulações computacionais. Foram adotados doze cenários para cada cidade e verificou-se que a captação de águas pluviais nos locais de estudo é viável para diminuir a demanda de água potável, contribuindo para o abastecimento descentralizado de água e o aumento da resiliência urbana. Por fim, entende-se que, quando agregada ao planejamento urbano, esta tecnologia tende a favorecer cidades menos vulneráveis hidricamente.
\end{abstract}

Palavras-chave: Política Hídrica; Vulnerabilidade Hídrica; Cidades Sensíveis à Água; Abastecimento Descentralizado; Cidades Resilientes.

ABSTRACT: Climate change and the increase in the urban population are strong stressors for water resources, making it necessary to discuss the construction of urban systems that are resilient and sensitive to water. In this way, the use of rainwater presents itself as an important alternative for accessing water and reducing pressure in water sources. This research aimed to identify the potential of rainwater harvesting in thirteen cities in the State of Paraíba by means of computer simulations. Twelve scenarios were adopted for each city and it was found that the capture of rainwater in the study sites is feasible to decrease the demand for drinking water, corroborating for the decentralized water supply and the increase of urban resilience. Finally, it is understood that, when added to urban planning, this technology tends to favor less hydric vulnerable cities.

Keywords: Water Policy; Water Vulnerability; Water-Sensitive Cities; Decentralized Supply; Urban Resilience.

\section{INTRODUÇÃo}

A escassez hídrica é proveniente não apenas de baixos índices pluviométricos, das mudanças climáticas e da falta de infraestrutura, mas, também, da deficiência ou ausência da governança da água (Pahl-Wostl, 2017) e de um ineficaz planejamento do território (Serrao-Neumann et al., 2017). A Organização para a Cooperação e Desenvolvimento Econômico (2015) entende que a governança da água representa o montante de regras, práticas e processos institucionais e administrativos, pelos quais as decisões são tomadas e implementadas. Já o planejamento urbano pode ser definido como "o conjunto de medidas tomadas para que sejam atingidos os objetivos desejados, tendo em vista os recursos disponíveis e os fatores externos que podem influenciar nesse processo" (Duarte, 2012, p. 26).

Serrao-Neumann et al. (2017) argumentam que esses dois fatores têm sido pensados e realizados de forma separada, mas que isso precisa ser alterado, promovendo integração para garantir

Recebido: Junho 02, 2021. Revisado: Agosto 27, 2021. Aceito: Setembro 28, 2021. 
sustentabilidade e cidades resilientes. A resiliência em um sistema pode ser entendida como a capacidade de absorver perturbações e se reorganizar conseguindo manter suas funções essenciais (Walker et al., 2004; Folke et al., 2010; Wilson et al., 2013; Jiménez et al., 2020).

As cidades são sistemas complexos em que há um conjunto de interações entre infraestruturas, o meio ecológico, aspectos socioculturais, dinâmicas econômicas e sistemas de governança (Zhang \& Li, 2018), portanto, faz-se necessário entender como esses espaços podem se tornar mais resilientes para responder a estresses. Para o projeto Smart Mature Resilience (2016, p. 52), cidade resiliente consiste na

capacidade de uma cidade ou região resistir, absorver, se adaptar e se recuperar de choques agudos e estresses crônicos para manter o funcionamento dos serviços críticos e monitorar e aprender com os processos em andamento por meio da colaboração municipal e interregional, para aumentar habilidades adaptativas e fortalecer a preparação antecipando e respondendo apropriadamente aos desafios futuros.

Sem a boa governança de águas e o planejamento urbano adequado, as mudanças climáticas e o aumento na demanda de recursos hídricos têm se configurado em fortes estressores para as cidades (Johannessen \& Wamsler, 2017; Iturriza et al., 2020), isto porque ameaça a garantia dos serviços de água e, consequentemente, a permanência das relações sistêmicas intrínsecas ao meio urbano. Nesse sentido, a resiliência opera para a manutenção de um bom nível de serviços de água ofertados, assim como, pela rápida recuperação das falhas causadas por uma gestão ineficaz, infraestruturas insuficientes ou desastres naturais, como os eventos de secas (Laitinen et al., 2020).

Com a aplicação do conceito do metabolismo urbano é possível entender que, não levando em consideração a gestão ambiental, inclusive dos recursos hídricos, no planejamento urbano, caminhase para problemas da ordem do esgotamento de recursos naturais, resultando em cidades vulneráveis e, portanto, menos resilientes (Marinho et al., 2020). Diante desse aspecto, faz-se necessário pensar e dialogar acerca de cidades sensíveis à água (Wong \& Brown, 2009), as quais planejam otimizar os recursos hídricos no meio urbano, importando menos água potável e exportando menos efluentes. E isto deve ser levado em consideração no planejamento urbano.

Alterar a lógica do abastecimento de água é um caminho que colabora com esse conceito de organização (Leigh \& Lee, 2019). 0 abastecimento centralizado é realidade em grande parte dos municípios brasileiros, isto é, praticamente toda demanda de água se apoia em um único corpo hídrico. Como quebra desse paradigma, diversos autores orientam para a necessidade de adotar múltiplas fontes de abastecimento (descentralizado ou híbrido), diminuindo a dependência de uma única fonte e, consequentemente, a vulnerabilidade dos centros urbanos em relação aos recursos hídricos (Sapkota et al., 2015; Chelleri et al., 2015; Ren et al., 2017; Leigh \& Lee, 2019; Marinho et al., 2020). Um entrave é percebido ao considerar que o marco legal do saneamento ao mesmo tempo que estabelece que os serviços públicos de saneamento devem estimular o reuso de efluentes e o aproveitamento de água de chuva também determina que "a instalação hidráulica predial ligada à rede pública de abastecimento de água não poderá ser também alimentada por outras fontes" (Brasil, 2007). Uma interpretação dessas informações é de que caberia às organizações responsáveis pelo abastecimento público a distribuição das outras fontes citadas na legislação (reuso de efluentes e água de chuva). Sendo, portanto, algo a ser questionado diante dos diversos benefícios que as fontes alternativas de água propiciam com a iniciativa individual.

São algumas fontes descentralizadas de água: o tratamento de esgoto e águas cinzas, a reutilização das águas da drenagem urbana e o armazenamento da água de chuva em residências (Sapkota et al., 2015; Leigh \& Lee, 2019). Cada uma dessas fontes apresenta potencialidade diferente, dessa forma, cabe aos municípios em suas distintas realidades a investigação de quais fontes podem ser adotadas para fortalecer a relação do meio urbano com os recursos hídricos.

Sabendo da necessidade de ampliar o conhecimento sobre a descentralização do abastecimento de água em cidades e da importante integração entre esta medida e o planejamento urbano, este artigo objetiva identificar a potencialidade de aproveitamento da água de chuva em residências de cidades do Estado da Paraíba, as quais estão sujeitos a diferentes condições hidrológicas e localizados em distintas regiões geográficas do estado. 0 foco se dá no aspecto ambiental e na resiliência urbana, utilizando uma metodologia de fácil replicabilidade para que outros municípios consigam entender o potencial de uso dessa fonte alternativa de água em seu domínio. 


\section{CAPTAÇÃO E APROVEITAMENTO DE ÁGUA DE CHUVA}

A gestão de recursos hídricos, um dos elementos que compõem a governança da água, no Brasil é pautada pela Lei 9.433/1997 (Política Nacional de Recursos Hídricos - PNRH), que estabelece a integração, a descentralização e a participação, com ações planejadas através da unidade de bacia hidrográfica. Em 2017, a partir da Lei 13.501/2017, foi acrescentado o objetivo IV à PNRH: "incentivar e promover a captação, a preservação e o aproveitamento de águas pluviais" (Brasil, 1997). Diante disso, a própria legislação reconhece a importância da utilização da água de chuva na gestão de demanda, visto que esta medida favorece a ampliação do acesso e reduz a exploração dos corpos hídricos.

Os reservatórios para captação de água de chuva têm um papel importante para a população brasileira que habita regiões com longos períodos de estiagem, como o semiárido, pois não somente contribuem para suprir os consumos básicos (Santos, 2008; Almeida, 2017), como também auxiliam em atividades econômicas, a exemplo da agricultura familiar (Ferreira, 2015).

0 aproveitamento de água de chuva é uma das medidas de convivência com a seca disseminadas pela Articulação no Semiárido Brasileiro (ASA). Dentre os programas desenvolvidos com esse objetivo citam-se: o Programa Um Milhão de Cisternas (P1MC) com o intuito de construir um milhão de cisternas de placas em áreas rurais, que captam água de chuva e garantem a água potável para as famílias, favorecendo a autonomia hídrica em tempos não chuvosos; o Programa Uma Terra e Duas Águas $(\mathrm{P} 1+2)$, que tem a finalidade de implementar tecnologias que captam e manejam água de chuva para garantir a dessedentação animal e irrigação; e o Cisternas nas Escolas, destinado às escolas rurais sem sistema de abastecimento regular de água potável, proporcionando segurança hídrica e alimentar (Articulação do Semiárido Brasileiro, 2019).

No Brasil, dentre as três principais escalas de planejamento (federal, estadual e municipal), fica a cargo dos estados e municípios desenvolver legislações específicas que auxiliem na difusão de tecnologias descentralizadas para o abastecimento de água (Libanio, 2014; Agência Nacional de Águas e Saneamento Básico, 2020). Leis que buscam o incentivo da prática de aproveitamento de água de chuva vêm sendo promulgadas, seja para conter inundações, seja para a adoção de práticas de consumo racional. Ward et al. (2019) citam a importância dos atos legislativos como incentivos e mostram como o Brasil tem começado a atuar positivamente nesse sentido. Exemplos desses instrumentos legais estaduais são: Lei 4.393/2004 (Rio de Janeiro), Lei 12.526/2007 (São Paulo), Lei 17.128/2010 (Goiás); Lei 9.130/2010 (Paraíba); Lei 18.730/2016 (Paraná). A nível municipal, são exemplos: Lei 2.256/2005 (Canela - RS); Lei 7.863/2010 (Salvador - BA); Lei 5.279/2011 (Rio de Janeiro - RJ); Lei 18.112/2015 (Recife - PE). Regulamentos, que fornecem regras claras, podem ser muito eficazes no apoio à instalação de sistemas descentralizados como os reservatórios para acumular água de chuva (Schuetze, 2013). Salientando, ainda, a importância de articulação entre políticas e programas já existentes, de modo a obter uma gestão eficiente das fontes alternativas de água (Nóbrega et al., 2012).

Em se tratando de parâmetros de qualidade da água de chuva e critérios construtivos de sistemas de captação, não existe uma legislação no nível nacional. Entretanto, há a Norma Brasileira (NBR) 15.527/2019 da Associação Brasileira de Normas Técnicas (ABNT) que apresenta definições para o aproveitamento de água de chuva para fins não potáveis e que é utilizada como balizadora para este mecanismo, apresentando, inclusive, alguns métodos de dimensionamento simplificados. Métodos estes que são considerados insuficientes quando comparados com programas computacionais que levam em consideração modelos comportamentais (Rupp et al., 2011).

Como exemplos da relevância do aproveitamento de água de chuva no cenário internacional, Kusre et al. $(2017,2018)$ consideram que a possibilidade de implantação de sistemas de captação de água de chuva no Estado de Sikkin, Índia, é uma oportunidade para aumentar a resiliência da comunidade e uma estratégia de gestão de recursos hídricos. Dismas et al. (2018) demonstram que a captação de água de chuva é uma alternativa para atenuar a escassez hídrica na cidade de Kinondoni, Tânzania, mas há a necessidade de participação integrada de distintos entes interessados em educar e apoiar as comunidades.

Em escala nacional, vários estudos têm reconhecido o potencial de utilização da água de chuva tanto no aspecto econômico como ambiental (Lopes et al., 2015; Souza, 2015). Na literatura é possível identificar a eficiência na utilização desse mecanismo nos mais diferentes tipos de construções. Porém, sabendo que a demanda de água em residências é bastante significativa (Agência Nacional de Águas e Saneamento Básico, 2018), muitos trabalhos se concentraram em analisar a potencialidade de uso dessa tecnologia social nesse tipo de edificação, como Sant'Ana (2019) em Brasília e Marinoski \& Ghisi (2019) em Santa Catarina. 
Em regiões com características mais críticas quanto à disponibilidade de água, Santos \& Farias (2017) analisaram o aproveitamento de água de chuva em 71 cidades do agreste de Pernambuco e constataram que a economia de água potável pode chegar a 25\%. Dias (2007), em análise realizada em João Pessoa (Paraíba), constatou que o aproveitamento de água de chuva é viável economicamente para residências de alto padrão. Em Campina Grande, Souza (2015) levou em consideração as limitações dos lotes para implantar os reservatórios para armazenamento de água de chuva e dessa forma percebeu que a cidade pode economizar até $11,35 \%$ de água potável, mas sua metodologia demanda dados georreferenciados de alta resolução que geralmente são de difícil acesso à maioria dos municípios brasileiros. Já Silva et al. (2020) também em Campina Grande estimaram que a economia de água potável pode chegar a 17,89\%, mas direcionaram a análise a um recente complexo habitacional e não a cidade por completo.

Percebe-se, ao analisar os estudos dispostos na literatura, que não há uma única metodologia para chegar ao potencial de economia de água potável fazendo uso de água de chuva e que algumas delas demandam informações não tão acessíveis para a realidade de muitos municípios.

Entendendo que existe o potencial de economizar água potável com a utilização de sistema de aproveitamento de água de chuva, faz-se necessário que as cidades incorporem essa medida no planejamento de modo a contribuir para sistemas mais resilientes. Nesse sentido, Lu \& Stead (2013) apresentam seis características que podem ser incluídas nesse processo de planejamento para alcançar resiliência nas cidades: atenção à situação atual; atenção às tendências como ameaças futuras; capacidade de envolver o público; capacidade de iniciar a ação; capacidade de definir metas; e capacidade de aprender com o estresse anterior. Planejamentos municipais que levem em consideração essas etapas no sentido de construir um espaço urbano que usufrua dos benefícios do aproveitamento de água de chuva tendem a se tornar menos vulneráveis hidricamente.

Programas podem incorporar tanto a conscientização da população acerca do armazenamento de água, como incentivos financeiros para adoção (Schuetze, 2013; Sheikh, 2020). Schuetze (2013) cita alguns programas que foram implementados de modo a facilitar a adoção dessa tecnologia em cidades alemãs, eles consistem em subsidiar $1 / 3$ dos custos totais dos materiais e obras de instalação, e empréstimos com juros reduzidos para viabilizar a implantação.

\section{METODOLOGIA}

\section{Área de estudo}

Esta pesquisa tem como área de estudo o Estado da Paraíba. O Estado é composto por 223 municípios e população de 3.766 .528 habitantes (Instituto Brasileiro de Geografia e Estatística, 2010b). A escolha pelo Estado se fundamentou nos resultados do Water Risk Atlas (World Resources Institute, 2019), que mostram que numa resolução temporal anual todos seus municípios apresentam risco hídrico mediano a alto, levando em consideração todo o conjunto de indicadores analisados (Figura 1b). Além disso, ao observar os boletins mensais do Monitor de Secas (2021) nota-se que na maior parte do tempo o Estado da Paraíba está condicionado a secas de moderadas a graves.

Pela classificação climática de Köppen-Geiger, a Paraíba está dividida em quatro tipos de clima: Aw (tropical quente e úmido, com chuvas no verão e seca no inverno), Am (característico de região monçônica), As (tropical quente e úmido com chuvas de outono-inverno) e Bsh (semiárido quente, com chuvas de verão e inverno seco) (Francisco \& Santos, 2017).

Foram escolhidas cidades de cada uma das onze bacias hidrográficas contidas na Paraíba, exceto da Guaju, pois nenhum dos 223 municípios está inserido completamente nela. Para escolha das cidades, primeiro, foram selecionados os pluviômetros que não continham falhas no monitoramento. Em seguida, escolheu-se o pluviômetro que apresentava um registro de dados mais longo e semelhante (em termos de médias mensais) aos postos vizinhos. Na Figura 1c o mapa de isoietas com médias de precipitações anuais apresenta como as chuvas tendem a variar espacialmente ao longo do Estado. Excetuando-se as cidades do litoral, todas as outras estão inseridas no semiárido brasileiro.

Como a bacia hidrográfica do Rio Paraíba tem grande dimensão e pode haver considerável variação no comportamento das chuvas em toda sua extensão, optou-se por escolher cidades na subbacia do Rio Taperoá, na Região do Médio Curso do Rio Paraíba e na Região do Baixo Curso do Rio Paraíba. Por esse mesmo motivo, para a bacia hidrográfica do Rio Piranhas foi adotada uma cidade na sub-bacia do Rio do Peixe e outra na sub-bacia do Rio Espinharas. A Figura 1a identifica, no mapa da Paraíba, cada cidade escolhida e em que bacia hidrográfica está localizada. 

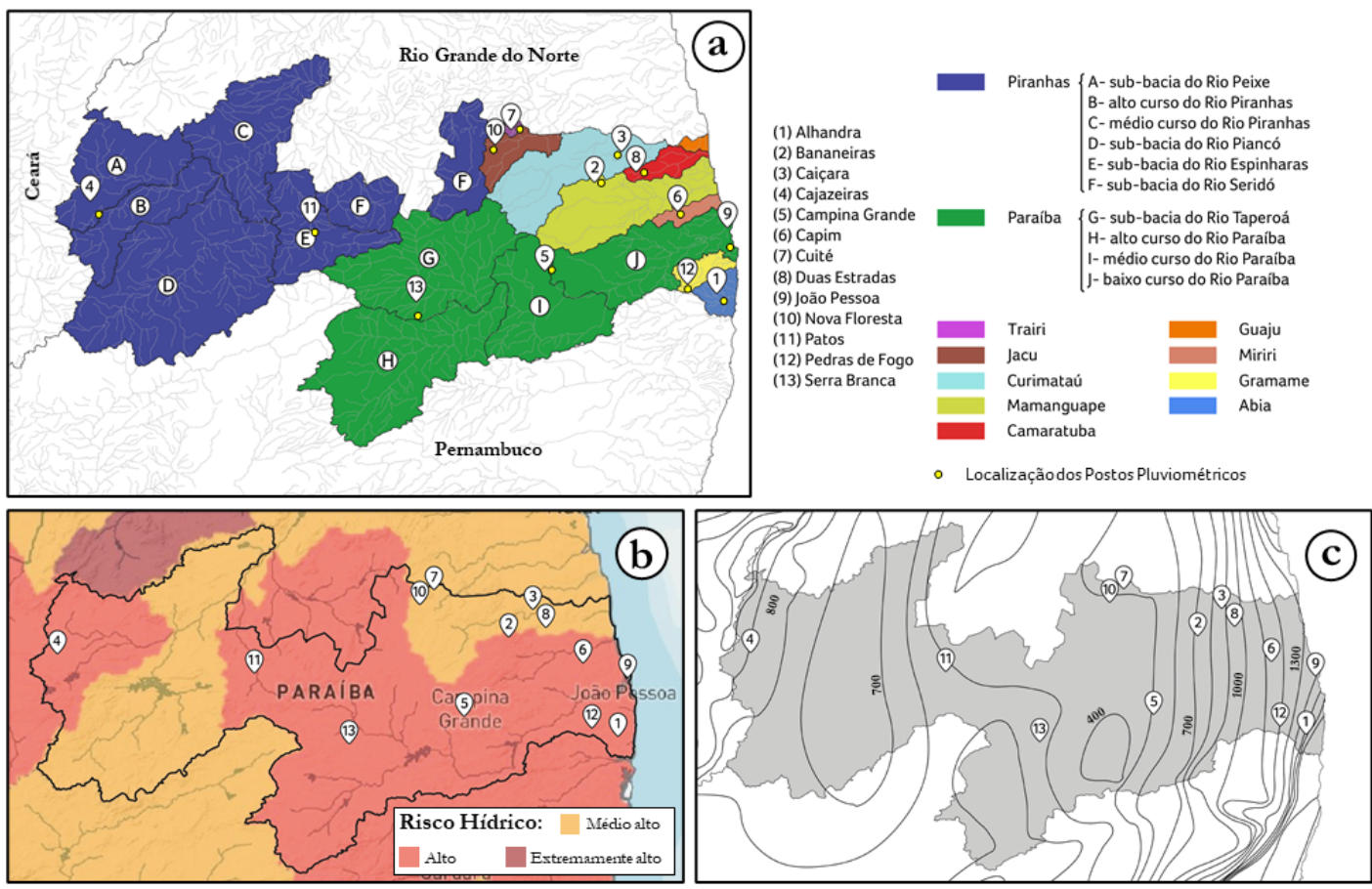

Figura 1 - Localização de cada cidade escolhida para o estudo (a) em sua respectiva bacia hidrográfica; (b) nas condições espacializadas de risco hídrico estabelecidas pelo Water Risk Atlas; (c) no mapa de isoietas com médias anuais em milímetros. Fonte: Elaborada pelos autores com malhas do Instituto Brasileiro de Geografia e

Estatística (2019), Agência Executiva de Gestão das Águas do Estado da Paraíba (2019), World Resources Institute (2019) e Agência Nacional de Águas e Saneamento Básico (2016).

\section{Procedimentos metodológicos}

Toda a metodologia e análises foram construídas para investigar o potencial de utilização de água de chuva para satisfazer as demandas não-potáveis em residências, reconhecendo as incertezas existentes sobre a qualidade desse recurso para outros fins mais nobres.

Para determinar a potencialidade de aproveitamento de água pluvial foi utilizado o Programa Netuno 4 (Ghisi \& Cordova, 2014), que tem uma interface simples e permite calcular o tamanho ideal para reservatórios que acumulam água de chuva e apresentar o potencial de economia de água potável. Essa ferramenta computacional utiliza modelos comportamentais (Fewkes, 2000) para realizar as simulações, isto é, simula o funcionamento do reservatório em relação ao tempo, observando como ocorre o fluxo de água através de um algoritmo que descreve a operação do reservatório. Teve sua eficiência validada por Rocha (2009) e foi usado como ferramenta em diversas pesquisas (Marinoski, 2010; Ribeiro, 2015; Ghisi et al., 2018; Cardoso et al., 2020). 0 potencial médio de economia de água potável e o volume ideal do reservatório são obtidos, respectivamente, dentre outras formulações pela Equação 1 e o algoritmo da Figura 2.

$E_{p o t}=100 * \sum_{i=1}^{N} \frac{V_{C}^{i}}{D_{t o t}^{i}}$

Em que Epot é o potencial de economia de água potável, em \%; $V_{C}^{i}$ é o volume de água de chuva consumido no dia i, em litros; e $D_{t o t}^{i}$ é a demanda total de água no dia i, em litros (Ghisi \& Cordova, 2014). 


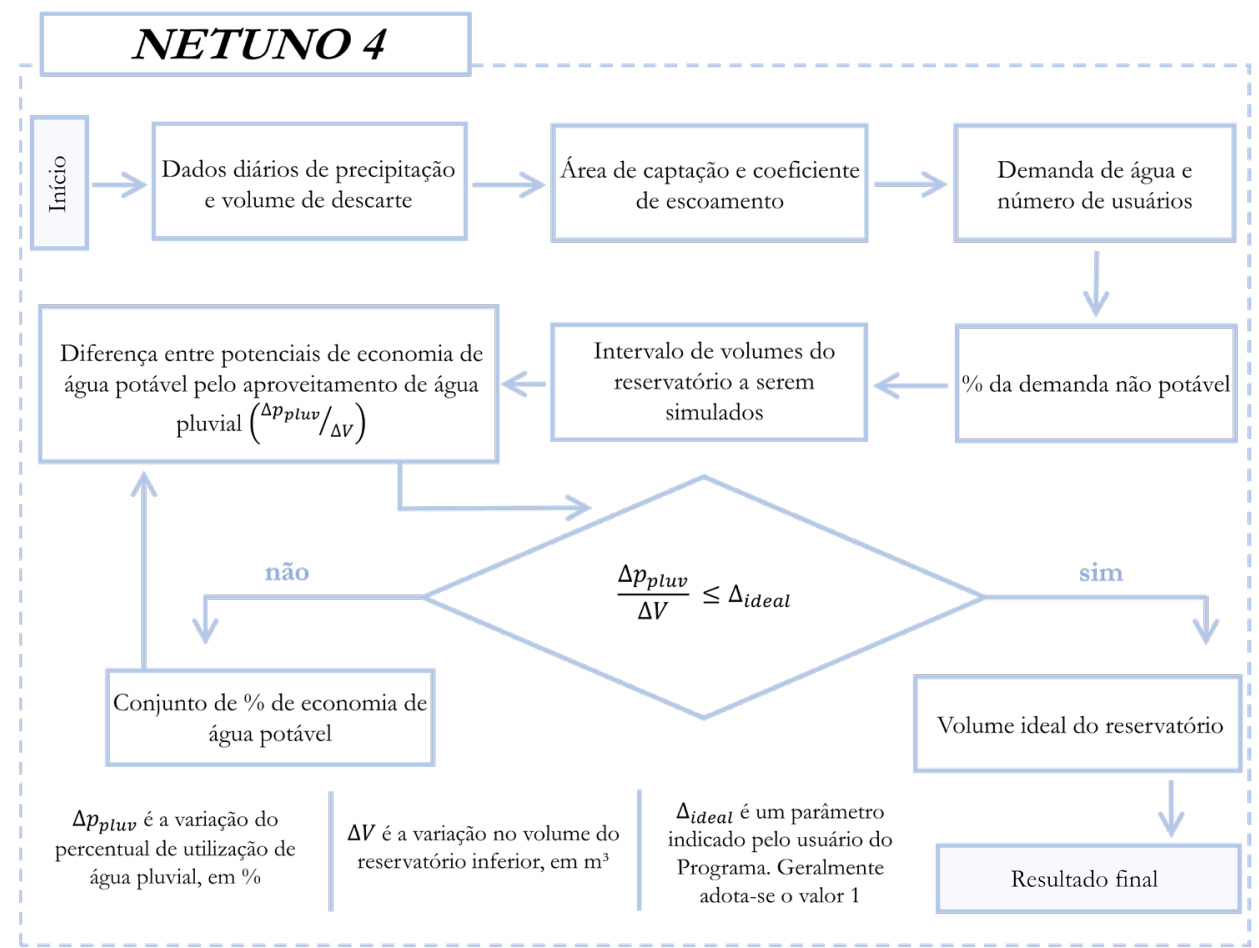

Figura 2 - Algoritmo do Programa Netuno 4 para definir o volume ideal do reservatório. Fonte: Adaptado de Cardoso et al. 2020.

Para gerar resultados, o programa necessita de uma série de dados de entrada, como apresentado na Figura 2: dados de precipitação, descarte do escoamento inicial (first-flush), área de captação, demanda de água per capita por dia, número de moradores por residência, percentual da demanda total a ser substituída por água pluvial e coeficiente de escoamento superficial.

Os dados pluviométricos necessários para as simulações foram obtidos no site da Agência Executiva de Gestão das Águas do Estado da Paraíba (2019). Nos municípios em que há mais de um posto pluviométrico, foi selecionado o que opera por mais tempo. Foram usadas séries temporais de chuvas diárias de onze anos, satisfazendo as orientações de Ghisi et al. (2012) e Geraldi \& Ghisi (2017), que esclarecem que séries com mais de dez anos oferecem confiança para as simulações. Para doze municípios o intervalo de dados utilizado foi de 2007 até 2017, para Cuité isso não foi possível devido a falhas no monitoramento de alguns anos. Logo optou-se por usar uma série consistente de 2004 até 2014 somente para este município. As precipitações anuais para cada posto analisado são apresentadas na Figura 3.

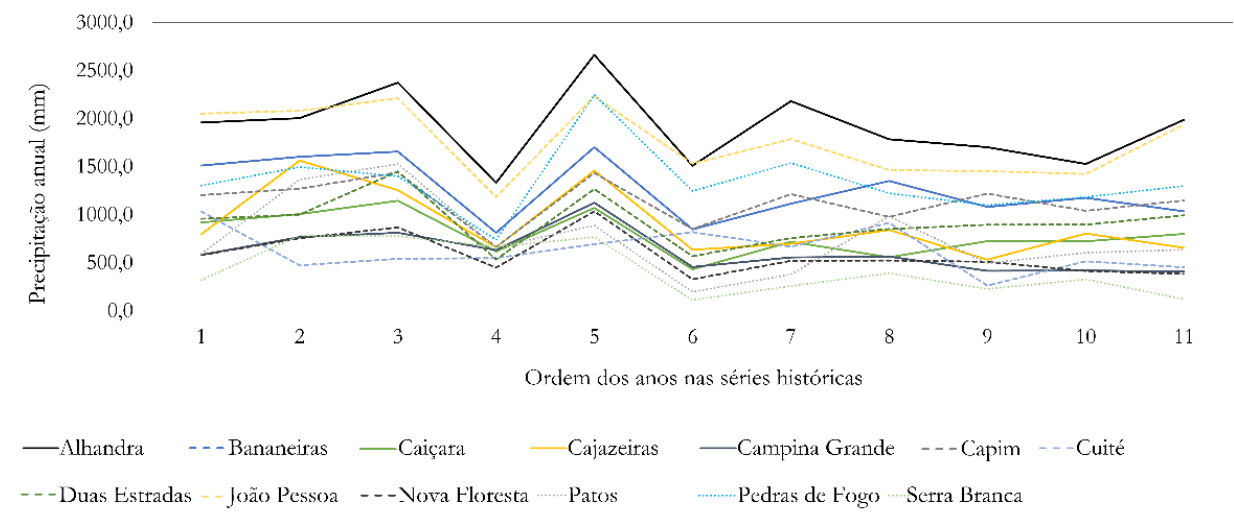

Figura 3 - Total precipitado para cada ano do período de análise para cada município. Fonte: Elaborada pelos autores com dados da Agência Executiva de Gestão das Águas do Estado da Paraíba (2019).

Admitiu-se o valor de $2 \mathrm{~mm}$ recomendado pela NBR 15.527 (Associação Brasileira de Normas Técnicas, 2019) como a quantidade de água a ser descartada inicialmente em cada precipitação (first- 
flush). Para o coeficiente de escoamento superficial foi adotado 0,85, por ser um valor intermediário dentre os que são comumente adotados (Rocha, 2009).

Os consumos per capita para cada uma das cidades escolhidas foram obtidos com as informações mais recentes no Sistema Nacional de Informação Sobre Saneamento (Sistema Nacional de Informações sobre Saneamento, 2019) no período de coleta de dados para as simulações (mês de junho de 2019) e são apresentados na Tabela 1. Os dados são referentes ao ano de 2017, com exceção das cidades de Cuité e Nova Floresta, que só dispunham de informações até o ano de 2014. Como os valores disponíveis para esse ano eram muito discrepantes dos anteriores, foram utilizados os valores de 2013. Além desses consumos, na Tabela 1, são incluídas informações como clima, população e número de habitantes por domicilio, entendendo que isso pode auxiliar em futuras comparações entre cidades com características semelhantes.

A demanda de água potável a ser substituída por água de chuva foi de $35 \%$ do consumo total, pois esse percentual se aproxima do valor utilizado no estudo feito por Marinoski etal. (2018), satisfazendo a condição de substituir um montante destinado para usos não potáveis. Esse percentual pode variar significativamente dentro de uma mesma cidade, pois depende, principalmente, dos tipos de equipamentos hidráulicos de cada residência e da dinâmica de uso dos moradores. Como exemplo disso, no estudo de Marinoski et al. (2018) nas residências analisadas (numa mesma cidade) o total de água destinado aos usos não potáveis nas residências variou entre 9\% e 50\%. Dessa forma, havendo esta limitação é comum na literatura que um percentual seja adotado e, aqui, foi utilizado o estudo citado por ter sido investigado em um número considerável de residências.

Tabela 1 - Informações sobre os municípios analisados.

\begin{tabular}{c|c|c|c|c}
\hline Cidade & População $*$ & $\begin{array}{c}\text { Número de } \\
\text { habitantes por } \\
\text { domicilio } * *\end{array}$ & $\begin{array}{c}\text { Clima (Köppen- } \\
\text { Geiger) }\end{array}$ & $\begin{array}{c}\text { Consumo per capita } \\
\text { (litros/hab.dia) }\end{array}$ \\
\hline Alhandra & 19.727 & 3,52 & Am & 116,09 \\
\hline Bananeiras & 21.269 & 3,53 & As & 71,79 \\
\hline Caiçara & 7.191 & 3,37 & As & 74,59 \\
\hline Cajazeiras & 62.289 & 3,33 & As & 89,39 \\
\hline Campina Grande & 411.807 & 3,42 & As e BSh & 100,31 \\
\hline Capim & 6.620 & 3,80 & As & 160,47 \\
\hline Cuité & 20.334 & 3,32 & BSh & 90,09 \\
\hline Duas Estradas & 3.582 & 3,30 & As & 107,38 \\
\hline João Pessoa & 817.511 & 3,37 & As & 155,84 \\
\hline Nova Floresta & 10.626 & 3,28 & BSh & 84,74 \\
\hline Patos & 108.192 & 3,46 & As & 110,20 \\
\hline Pedras de Fogo & 28.533 & 3,53 & BSh & 110,03 \\
\hline Serra Branca & 13.754 & 3,11 & 95,05
\end{tabular}

* População estimada pelo IBGE para o ano de 2020, levando em consideração a área urbana e rural, ** Número de habitantes por domicilio calculado com informações do censo realizado pelo IBGE em 2010 e levando em consideração somente a população urbana. Fonte: Elaborada pelos autores com dados do Instituto Brasileiro de Geografia e Estatística (2010a, 2020), Francisco \& Santos (2017) e Sistema Nacional de Informações sobre Saneamento (2019).

Inicialmente, utilizou-se a condição de que o volume máximo que os reservatórios poderiam assumir seria de 15.000 litros. Isso admitindo que armazenadores para quantidade de água superior a esta necessitam de espaços consideráveis para sua implantação, algo que é difícil na maioria das residências urbanas (Souza, 2015).

As simulações foram feitas para residências com área de captação de 50, 100, 200 e 300 m², pois são valores próximos aos adotados por Dias (2007), Lopes et al. (2015) e Santos \& Farias (2017), e com número de moradores variando entre 2 e 4, que são valores aproximados à média de moradores por domicílio na Paraíba como um todo (3,41 habitantes por domicilio) (Instituto Brasileiro de Geografia e Estatística, 2010a) e nas cidades escolhidas (Tabela 1). Dessa forma foram realizadas 156 simulações. Para cada município fixouse a série histórica de chuva correspondente e variou-se a área de captação da residência e o número de moradores, o que resultou em doze cenários para cada uma das treze cidades (para cada área de captação, simulou-se com 2, 3 e 4 moradores). Dentre os cenários simulados há o mais positivo (residência com $300 \mathrm{~m}^{2}$ e 2 moradores) e o mais crítico (residência com $50 \mathrm{~m}^{2}$ e 4 moradores).

0 percentual de utilização de água de chuva foi calculado pela Equação 2. Esse indicador corresponde a razão entre o potencial de economia de água potável alcançado (resultado das simulações) e o valor 
estabelecido de água potável a ser substituído por água de chuva (neste estudo, 35\% do total). Portando, demonstra quanto do volume almejado de água de chuva foi atendido no período simulado.

$\mathrm{P}_{\text {pluv }}=\frac{E_{\text {pot }}}{p_{\text {subs }}}$

Onde, Ppluv é o percentual de utilização de água de chuva; Epot é potencial de economia de água potável por meio do aproveitamento da água de chuva em porcentagem; Psubs é percentual de água potável substituída por água de chuva (Ghisi \& Cordova, 2014).

\section{RESULTADOS E DISCUSSÕES}

A síntese dos resultados das simulações executadas no programa Netuno 4 (Ghisi \& Cordova, 2014) para as cidades em estudo neste trabalho é exposta na Tabela 2.

Tabela 2 - Síntese dos resultados das simulações realizadas no Programa Netuno 4.

\begin{tabular}{|c|c|c|c|c|c|}
\hline \multirow{2}{*}{ Cidade } & \multicolumn{2}{|c|}{$\begin{array}{l}\text { Volume ideal dos reservatórios } \\
\text { (litros) }\end{array}$} & \multirow{2}{*}{ Falhas } & \multirow{2}{*}{$\begin{array}{c}\text { Potencial } \\
\text { médio de } \\
\text { economia de } \\
\text { água potável } \\
(\%) \\
\end{array}$} & \multirow{2}{*}{$\begin{array}{c}\text { Percentual } \\
\text { médio de } \\
\text { utilização de } \\
\text { água de chuva } \\
\text { (\%) }\end{array}$} \\
\hline & Mínimo & Máximo & & & \\
\hline Alhandra & 3000 & 15000 & 0 & 34,5 & 98,57 \\
\hline Bananeiras & 3750 & 11750 & 0 & 34,54 & 98,69 \\
\hline Caiçara & 4500 & 12500 & 0 & 33,26 & 95,03 \\
\hline Cajazeiras & 9500 & 14750 & 5 & 33,17 & 94,77 \\
\hline Campina Grande & 3750 & 14500 & 2 & 28,96 & 82,74 \\
\hline Capim & 6000 & 13000 & 5 & 31,12 & 88,91 \\
\hline Cuité & 7000 & 14250 & 3 & 31,13 & 88,94 \\
\hline Duas Estradas & 7250 & 14500 & 1 & 32,69 & 93,40 \\
\hline João Pessoa & 5250 & 14500 & 3 & 34,01 & 97,17 \\
\hline Nova Floresta & 6750 & 13250 & 3 & 30,77 & 87,91 \\
\hline Patos & 9250 & 14250 & 8 & 26,25 & 75,00 \\
\hline Pedras de Fogo & 4000 & 14000 & 1 & 34,34 & 98,11 \\
\hline Serra Branca & 4750 & 15000 & 1 & 25,05 & 71,57 \\
\hline
\end{tabular}

A partir da Tabela 2 é possível identificar que as cidades que necessitam de reservatórios de menores capacidades de armazenamento são Alhandra e Bananeiras, isso porque apresentam índices pluviométricos anuais maiores que os da maioria (Figura 2). Ressalta-se que, embora a cidade de João Pessoa tenha maiores valores de precipitação anuais entre as cidades em análise, ela não reproduz a situação de Alhandra e Bananeiras, pois apresenta o consumo per capita bem mais elevado, o segundo maior dentre as cidades avaliadas, necessitando, portanto, acumular mais água para suprir sua demanda. Esse fator é um dos que dificulta correlacionar a classificação de clima de Köppen-Geiger com os volumes, pois mesmo que as precipitações sejam semelhantes nas regiões definidas por Francisco \& Santos (2017), o consumo per capita fará com que existam discrepâncias consideráveis nos volumes dos reservatórios.

Dez das treze cidades apresentaram falhas ao se proceder a modelagem visando estabelecer o volume ideal do reservatório. Isso ocorreu porque foi estabelecido um volume máximo de 15.000 litros e o programa não encontrou reservatório ideal com volume inferior ou igual a esse. Essa ação foi entendida como falhas no processo de simulação e, portanto, o potencial de economia de água potável não foi computado para os cenários em que isso ocorreu.

Patos foi a cidade que apresentou mais falhas, isto é, ausência de especificação da capacidade ideal do reservatório, atendendo apenas quatro dos doze cenários. Este fator está relacionado não só ao consumo per capita e à quantidade de chuva, mas também à forma como a precipitação se distribui ao longo do ano. Dentre as cidades que apresentaram o maior número de falhas, Patos e Cajazeiras estão situadas na bacia do Rio Piranhas e possuem um total de precipitação anual superior ao de cidades como Serra Branca e Nova Floresta. Em contrapartida, esse volume varia muito ao longo dos meses, como é mostrado na Figura 4, sendo necessários reservatórios maiores para suprir as demandas nos períodos do ano com menor precipitação. 


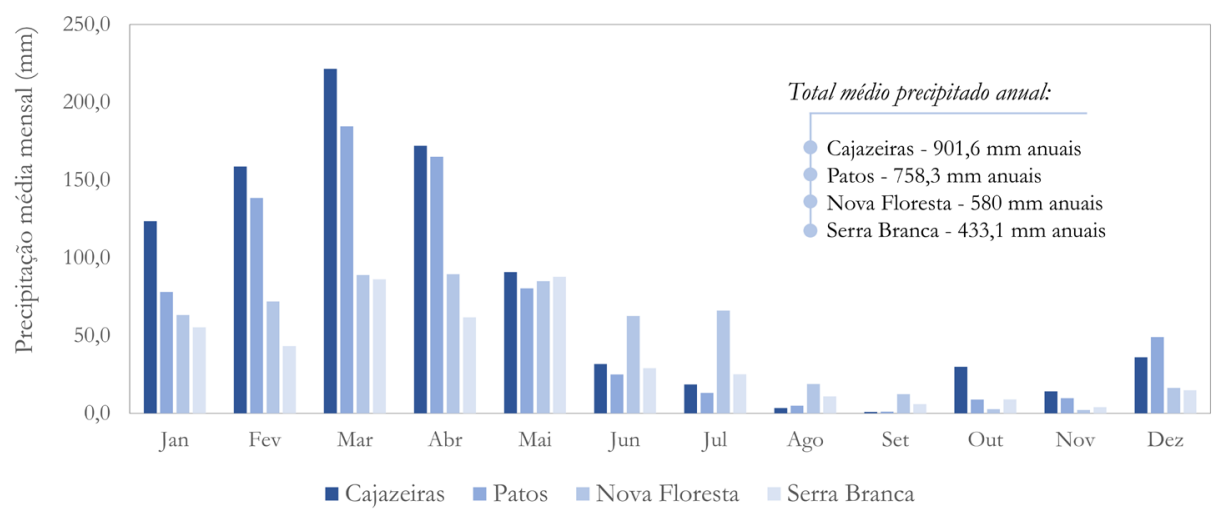

Figura 4 - Média de precipitação nos doze meses do ano para as cidades de Patos, Cajazeiras, Nova Floresta e Serra Branca. Fonte: Elaborada pelos autores com dados da Agência Executiva de Gestão das Águas do Estado da Paraíba (2019).

As cidades com maior quantidade de falhas ou menor volume de chuvas apresentam piores resultados quanto ao potencial médio de economia de água potável e, consequentemente, ao percentual médio de utilização de água pluvial (Equação 2), visto que seriam necessários reservatórios maiores ou precipitações mais regulares para atender toda a demanda de água de chuva ao longo do ano.

Analisar o percentual de utilização de água pluvial é importante porque esse parâmetro permite determinar o quanto da demanda por água de chuva será satisfeita, auxiliando na gestão dos recursos hídricos para o abastecimento e fortalecendo a governança. Das cidades analisadas, Serra Branca, Patos e Campina Grande, são as que menos conseguem substituir o consumo de água potável com a utilização de água pluvial. A primeira possui essa deficiência associada aos fatores meteorológicos, a segunda pelos motivos já citados nesta pesquisa, e a terceira em razão do consumo per capita, que é um dos maiores dentre todas as cidades analisadas, e aos baixos índices pluviométricos.

Um dos fatores relacionados ao baixo potencial de resultados satisfatórios em algumas cidades é o consumo, como já mencionado. Logo, pode-se propor uma gestão de demanda de água fazendo uso, por exemplo, de equipamentos poupadores como bacias sanitárias com duplo acionamento. Essa ação tem o intuito de reduzir a quantidade de água requerida pelas pessoas, o que resultaria na necessidade de um menor volume de água de chuva para suprir os usos não potáveis.

Percebeu-se que muitos dos volumes ideais resultantes das simulações são maiores que os atribuídos em pesquisas para outras cidades brasileiras (Chaib et al., 2015; Marinoski et al., 2018). Muitos podem ser os condicionantes para essa constatação, dentre eles o regime de precipitações como já mencionado, pois muitas dessas cidades estão situadas no semiárido brasileiro, o que as submete a severos fenômenos de seca.

Sabendo que quanto maiores os reservatórios, maior a limitação urbanística nos lotes e maior o custo de implantação, coube também avaliar o aproveitamento de água de chuva para volumes comerciais e mais reduzidos dos armazenadores. Souza (2015) apresenta os volumes de reservatórios que podem ser implantados em Campina Grande levando em consideração as restrições urbanísticas dos lotes. Desta forma, com um cenário usando reservatórios de volume comercial até $10 \mathrm{~m}^{3}$, o autor mostra que de todos os lotes analisados: 80,4\% comporta reservatórios de 10.000 litros; 3,9\% comporta reservatórios de 5.000 litros; 0,6\% comporta reservatórios de 1.000 litros e $15 \%$ comporta reservatórios de 500 litros.

Na Figura 5 são apresentados os resultados para simulações com esses quatro volumes de reservatórios mais reduzidos levando em consideração o cenário mais crítico (residência com $50 \mathrm{~m}^{2}$ e 4 moradores) e o cenário mais positivo (residência com $300 \mathrm{~m}^{2}$ e 2 moradores). Todos os outros cenários têm resultados entre estes. Percebe-se que a grande maioria das cidades, mesmo com reservatórios pequenos de 500 litros, consegue ter uma economia de água potável próxima ou superior a $10 \%$. Isso demonstra a potencialidade dessa tecnologia para racionalizar o uso da água e descentralizar o abastecimento, pressionando menos os corpos hídricos e, portanto, diminuindo possíveis estresses a esse recurso fundamental para as relações sistêmicas do meio urbano.

É importante ressaltar que quanto menor o volume do reservatório, e por mais que se consiga ter uma economia considerável de água potável, menor é o atendimento da água de chuva para satisfazer as demandas não potáveis. Portanto, faz-se necessário um balanço entre esses dois fatores ao planejar a implementação desses sistemas. 

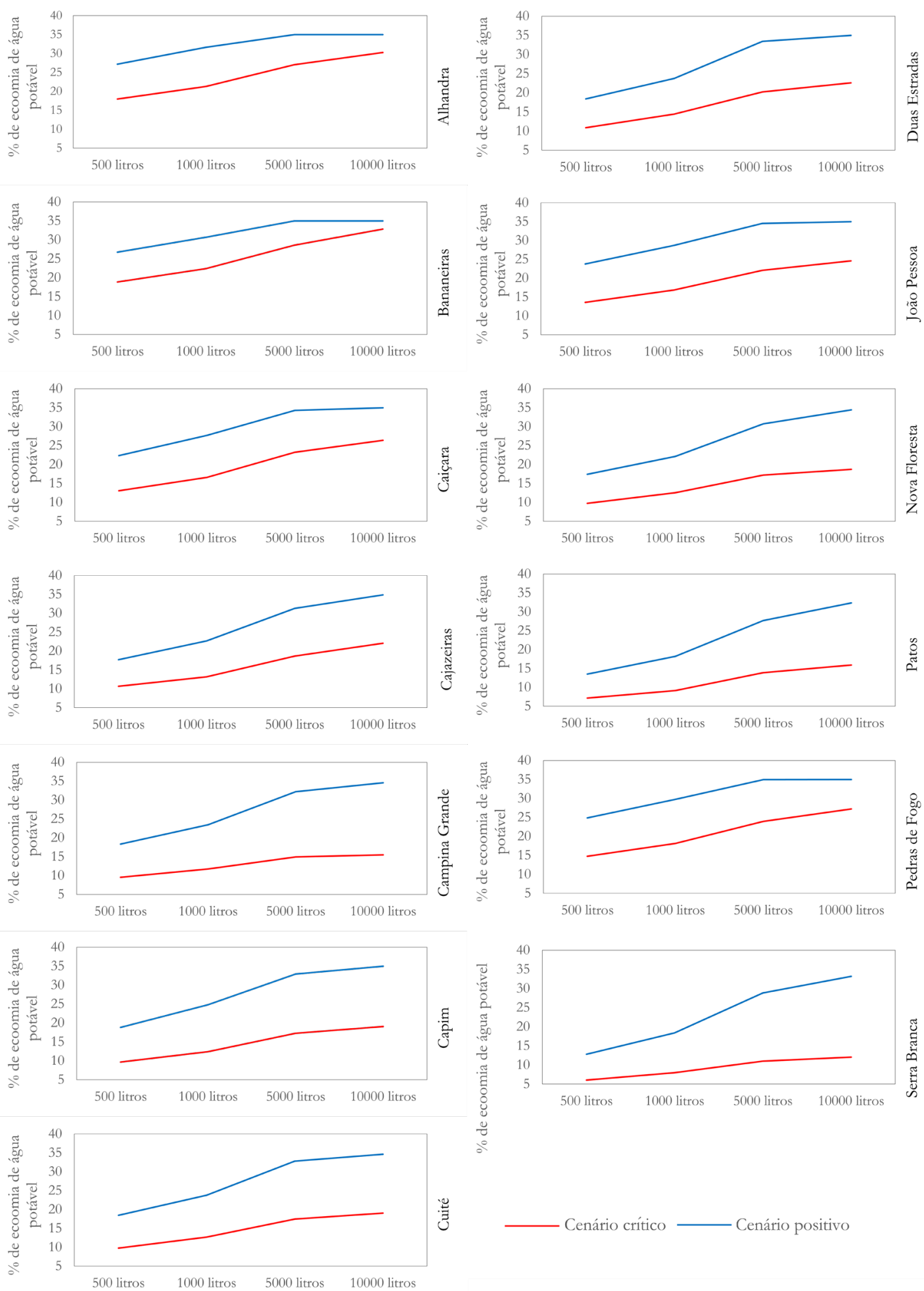

Cenário crítico - Cenário positivo

Figura 5 - Percentual de economia de água potável para diferentes reservatórios levando em consideração o cenário mais crítico (residência com $50 \mathrm{~m}^{2} \mathrm{e} 4$ moradores) e o mais positivo (residência com $300 \mathrm{~m}^{2}$ e 2 moradores).

\section{CONCLUSÕES}

A partir de simulações efetuadas através do programa Netuno 4, constatou-se que a captação e a utilização de águas pluviais em regiões urbanas apresentam-se viáveis para as cidades da Paraíba analisadas neste trabalho. Alcançou-se um percentual de redução de consumo de outras fontes (economia de água potável) de até 34,54\%, embora em alguns casos sejam necessárias outras medidas complementares em conjunto. 
Observou-se, ainda, que a distribuição das chuvas, o consumo per capita e o tamanho da cobertura são fatores relevantes para o dimensionamento de reservatórios e para o potencial de redução de consumo de água proveniente dos corpos hídricos. Quanto maior a variação mensal da pluviometria, maiores também deverão ser os reservatórios para acumulação. Mesmo que haja volumes de chuvas razoáveis, se o consumo per capita for elevado, a capacidade dos reservatórios também deve ser maior.

Destaca-se que não é possível caracterizar o potencial de captação de água de chuva para as bacias hidrográficas estudadas neste artigo em sua integralidade utilizando apenas as cidades de referência, pois em uma mesma bacia, cidades que apresentam volumes precipitados semelhantes podem apresentar valores de consumo per capita bastante distintos, alterando os volumes ideais dos reservatórios e a potencialidade da utilização da água de chuva.

Por fim, a pesquisa reafirma a capacidade do aproveitamento de água de chuva como tecnologia fortalecedora para o abastecimento descentralizado no meio urbano e demonstra ser benéfico, inclusive, em cidades de clima semiárido e utilizando reservatórios com volumes bastante reduzidos. Essas constatações contribuem para incentivar um planejamento urbano integrado com a governança de águas, para o fortalecimento das políticas de recursos hídricos e para a construção de cidades mais resilientes, portanto, menos vulneráveis hidricamente.

\section{AGRADECIMENTOS}

Os dois primeiros autores agradecem à Coordenação de Aperfeiçoamento de Pessoal de Nível Superior (CAPES) pelas bolsas de estudos concedidas.

\section{REFERÊNCIAS}

Agência Executiva de Gestão das Águas do Estado da Paraíba - AESA. (2019). Meteorologia: chuvas. Recuperado em 22 de abril de 2019, de http://www.aesa.pb.gov.br/aesa-website/meteorologia-chuvas/

Agência Nacional de Águas e Saneamento Básico - ANA. (2016). Catálogo de metadados da ANA. Recuperado em 17 de agosto de 2021, de https://metadados.snirh.gov.br/geonetwork/srv/api/records/befdedc6-d7534f09-a309-8957d7fe5f61

Agência Nacional de Águas e Saneamento Básico - ANA. (2018). Conjuntura dos recursos hídricos no Brasil. Brasília: ANA. Recuperado em 13 de março de 2019, de http://conjuntura.ana.gov.br/

Agência Nacional de Águas e Saneamento Básico - ANA. (2020). Direito de águas à luz da governança. Brasília: ANA.

Almeida, C. L. (2017). A convivência com o semiárido a partir do uso de cisternas de placas na zona rural do município de Frecheirinha-CE: dimensões na paisagem da superfície sertaneja (Dissertação de mestrado). Universidade Estadual Vale do Acaraú, Sobral.

Articulação do Semiárido Brasileiro - ASA. (2019). Semiárido Caderno de Debates - Caderno 01: propostas da sociedade civil para a garantia do acesso à água às populações rurais do Semiárido. Recuperado em 20 de abril de 2020, de https://www.yumpu.com/pt/document/read/62697726/frente-parlamentar-propostasda-sociedade-civil-para-a-garantia-do-acesso-a-agua-as-populacoes-rurais-do-semiarido

Associação Brasileira de Normas Técnicas - ABNT. (2019). NBR 15527: água de chuva: aproveitamento de coberturas em áreas urbanas para fins não potáveis: requisitos (2. ed.). Rio de Janeiro: ABNT.

Brasil. (1997, 8 de janeiro). Lei n. 9.433, de 8 de janeiro de 1997. Institui a Política Nacional de Recursos Hídricos. Diário Oficial [da] República Federativa do Brasil, Brasília.

Brasil. (2007, 5 de janeiro). Lei n. 11.445, de 5 de janeiro de 2007. Estabelece as diretrizes nacionais para o saneamento básico. Diário Oficial [da] República Federativa do Brasil, Brasília.

Cardoso, R. N. C., Blanco, C. J. C., \& Duarte, J. M. (2020). Technical and financial feasibility of rainwater harvesting systems in public buildings in Amazon, Brazil. Journal of Cleaner Production, 260, 121054.

Chaib, E. B., Rodrigues, F. C., Maia, B. H., \& Nascimento, N. O. (2015). Avaliação do potencial de redução do consumo de água potável por meio da implantação de sistemas de aproveitamento de água de chuva em edificações unifamiliares. Revista Brasileira de Recursos Hídricos, 20(3), 605-614.

Chelleri, L., Schuetze, T., \& Salvati, L. (2015). Integrating resilience with urban sustainability in neglected neighborhoods: challenges and opportunities of transitioning to decentralized water management in Mexico City. Habitat International, 48, 122-130. 
Dias, I. C. S. (2007). Estudo da viabilidade técnica, econômica e social do aproveitamento de água de chuva em residências na cidade de João Pessoa (Dissertação de mestrado). Universidade Federal da Paraíba, João Pessoa.

Dismas, J., Mulungu, D. M. M., \& Mtalo, F. W. (2018). Advancing rainwater harvesting as a strategy to improve water access in Kinondoni municipality, Tanzania. Water Science and Technology: Water Supply, 18(3), 745753.

Duarte, F. (2012). Planejamento urbano. Curitiba: InterSaberes.

Ferreira, E. P. (2015). Manejo da água de cisterna para a produção de alimentos no semiárido pernambucano (Dissertação de mestrado). Universidade Federal Rural de Pernambuco, Recife.

Fewkes, A. (2000). Modelling the performance of rainwater collection systems: towards a generalised approach. Urban Water, 1(4), 323-333.

Folke, C., Carpenter, S. R., Walker, B., Scheffer, M., Chapin, T., \& Rockström, J. (2010). Resilience thinking: integrating resilience, adaptability and transformability. Ecology and Society, 15(4), 1-9.

Francisco, P. R. M., \& Santos, D. (2017). Climatologia do Estado da Paraíba. Campina Grande: Edufcg.

Geraldi, M. S., \& Ghisi, E. (2017). Influence of the length of rainfall time series on rainwater harvesting systems: a case study in Berlin. Resources, Conservation and Recycling, 125, 169-180.

Ghisi, E., \& Cordova, M. M. (2014). Netuno 4: programa computacional. Recuperado em 31 de maio de 2019, de http://www.labeee.ufsc.br/

Ghisi, E., Cardoso, K. A., \& Rupp, R. F. (2012). Short-term versus long-term rainfall time series in the assessment of potable water savings by using rainwater in houses. Journal of Environmental Management, 100, 109-119.

Ghisi, E., Thives, L. P., \& Paes, R. F. W. (2018). Investment feasibility analysis of rainwater harvesting in a building in Brazil. Water Science and Technology: Water Supply, 18(4), 1497-1504.

Instituto Brasileiro de Geografia e Estatística - IBGE. (2010a). Média de moradores em domicílios particulares ocupados. Rio de Janeiro: IBGE. Recuperado em 20 de abril de 2019, de https://sidra.ibge.gov.br/tabela/3451

Instituto Brasileiro de Geografia e Estatística - IBGE. (2010b). População residente, por sexo e situação do domicílio da Paraíba. Rio de Janeiro: IBGE. Recuperado em 21 de abril de 2019, de https://sidra.ibge.gov.br/Tabela/202

Instituto Brasileiro de Geografia e Estatística - IBGE. (2019). Downloads. Rio de Janeiro: IBGE. Recuperado em 15 de maio de 2021, de https://downloads.ibge.gov.br/

Instituto Brasileiro de Geografia e Estatística - IBGE. (2020). Cidades. Rio de Janeiro: IBGE. Recuperado em 20 de stembro de 2020, de https://cidades.ibge.gov.br/

Iturriza, M., Labaka, L., Hernantes, J., \& Abdelgawad, A. (2020). Shifting to climate change aware cities to facilitate the city resilience implementation. Cities, 101, 1-11.

Jiménez, M., Pérez-Belmont, P., Schewenius, M., Lerner, M., \& Mazari-Hiriart, M. (2020). Assessing the historical adaptive cycles of an urban social-ecological system and its potential future resilience: the case of xochimilco, mexico city. Regional Environmental Change, 20(7), 1-14.

Johannessen, Å., \& Wamsler, C. (2017). What does resilience mean for urban water services? Ecology and Society, 22(1), 1-18.

Kusre, B. C., Bora, P. K., Rai, D., Adhikari, K., Niranjit Khuman, C., \& Tabing, J. (2018). Developing water resources management strategies for South Sikkim District, India. Water Science and Technology: Water Supply, 18(2), 648-659.

Kusre, B. C., Bora, P. K., Rai, D., Suranjoy, S. S., Tana, A., \& Subba, S. (2017). Combating water scarcity through roof water harvesting: planning and design with stakeholders' perception in Sikkim (India). Water Science and Technology: Water Supply, 17(3), 799-810.

Laitinen, J., Kallio, J., Katko, T. S., Hukka, J. J., \& Juuti, P. (2020). Resilient urban water services for the 21th century society: stakeholder survey in Finland. Water, 12(1), 1-12.

Leigh, N. G., \& Lee, H. (2019). Sustainable and resilient urban water systems: the role of decentralization and planning. Sustainability, 11(3), 2-17.

Libanio, P. A. C. (2014). The use of goal-oriented strategies in the building of water governance in Brazil. Water International, 39(4), 401-416. 
Lopes, A. C., Rupp, R. F., \& Ghisi, E. (2015). Assessment of the potential for potable water savings by using rainwater in houses in southern Brazil. Water Science and Technology: Water Supply, 16(2), 533-541.

Lu, P., \& Stead, D. (2013). Understanding the notion of resilience in spatial planning: a case study of Rotterdam, The Netherlands. Cities, 35, 200-212.

Marinho, S. D. A. M., Galvão, C. O., \& Miranda, L. I. B. (2020). A cidade sensível à água sob a perspectiva do metabolismo urbano e da análise da produção do espaço. Engenharia Sanitaria e Ambiental, 25(5), 1-11.

Marinoski, A. K. (2010). Método para avaliação de viabilidade ambiental e econômica de sistemas de aproveitamento de água pluvial (Dissertação de mestrado). Universidade Federal de Santa Catarina: Florianópolis.

Marinoski, A. K., \& Ghisi, E. (2019). Environmental performance of hybrid rainwater-greywater systems in residential buildings. Resources, Conservation and Recycling, 144, 100-114.

Marinoski, A. K., Rupp, R. F., \& Ghisi, E. (2018). Environmental benefit analysis of strategies for potable water savings in residential buildings. Journal of Environmental Management, 206, 28-39.

Monitor de Secas. (2021). Monitor de secas. Recuperado em 26 de agosto de 2021, de http://monitordesecas.ana.gov.br/mapa?mes=7\&ano=2021.

Nóbrega, R. L. B., Galvão, C. O., Palmier, L. R., \& Ceballos, B. S. O. (2012). Aspectos Político-Institucionais do Aproveitamento de Água de Chuva em Áreas Rurais do Semiárido Brasileiro. Revista Brasileira de Recursos Hídricos, 17(4), 109-124.

Organização para a Cooperação e Desenvolvimento Econômico - OCDE. (2015). OECD principles on water governance welcomed by ministers at the OECD Ministerial Council Meeting on 4 June 2015. Paris: OECD. Recuperado em 10 de maio de 2019, de https://www.oecd.org/cfe/regional-policy/OECD-Principles-onWater-Governance.pdf

Pahl-Wostl, C. (2017). An evolutionary perspective on water governance: from understanding to transformation Water Resources Management, 31(10), 2917-2932.

Ren, N., Wang, Q., Wang, Q., Huang, H., \& Wang, X. (2017). Upgrading to urban water system 3.0 through sponge city construction. Frontiers of Environmental Science \& Engineering, 11(9), 1-8.

Ribeiro, A. K. M. (2015). Método para avaliação do impacto ambiental da implantação de sistemas integrados de aproveitamento de água pluvial e água cinza em residências unifamiliares a partir da análise do ciclo de vida (Tese de doutorado). Universidade Federal de Santa Catarina, Florianópolis.

Rocha, V. L. (2009). Validação do algoritmo do programa netuno para avaliação do potencial de economia de água potável e dimensionamento de reservatórios de sistemas de aproveitamento de água pluvial em edificações (Dissertação de mestrado). Universidade Federal de Santa Catarina, Florianópolis.

Rupp, R. F., Munarim, U., \& Ghisi, E. (2011). Comparação de métodos para dimensionamento de reservatórios de água pluvial. Ambiente Construído, 11(4), 47-64.

Sant'Ana, D. (2019). Rainwater harvesting in Brazil: investigating the viability of rainwater harvesting for a household in Brasília. In G. Broadbent \& C. A. Brebbia (Eds.), Eco-architecture: harmonisation between architecture and nature (pp. 381-390). Southamption: WIT Press.

Santos, C. S. M. (2008). Avaliação do desempenho das cisternas de placas para abastecimento humano de água em comunidades rurais no semi-árido brasileiro (Dissertação de mestrado). Universidade Federal do Rio de Janeiro, Rio de Janeiro.

Santos, S. M., \& Farias, M. M. M. W. E. C. (2017). Potential for rainwater harvesting in a dry climate: assessments in a semiarid region in northeast Brazil. Journal of Cleaner Production, 164, 1007-1015.

Sapkota, M., Arora, M., Malano, H., Moglia, M., Sharma, A., George, B., \& Pamminger, F. (2015). An overview of hybrid water supply systems in the context of urban water management: challenges and opportunities. Water, 7(1), 153-174.

Schuetze, T. (2013). Rainwater harvesting and management: policy and regulations in Germany. Water Science and Technology: Water Supply, 13(2), 376-385.

Serrao-Neumann, S., Renouf, M., Kenway, S. J., \& Choy, D. L. (2017). Connecting land-use and water planning: prospects for an urban water metabolism approach. Cities, 60(A), 13-27.

Sheikh, V. (2020). Perception of domestic rainwater harvesting by Iranian citizens. Sustainable Cities and Society, 60,102278 
Silva, M. B. M., Dantas, A. K. E. A., \& Rodrigues, A. C. L. (2020). Captação de águas pluviais para o aumento da resiliência e desenvolvimento de um desenho urbano sensível à água. Revista Nacional de Gerenciamento de Cidades, 8(59), 35-52.

Sistema Nacional de Informações sobre Saneamento - SNIS. (2019). SNIS: série histórica. Brasília: SNIS. Recuperado em 20 de junho de 2019, de http://app4.mdr.gov.br/serieHistorica/\#

Smart Mature Resilience. (2016). Deliverable 1.3: about smart mature resilience. Recuperado em 27 de janeiro de 2021, de https://smr-project.eu/fileadmin/user_upload/Documents/Resources/WP_1/D1.3.SMR_Final.pdf

Souza, T. J. (2015). Potencial de Aproveitamento de Água de Chuva no Meio Urbano: o caso de Campina Grande PB (Dissertação de mestrado). Universidade Federal de Campina Grande, Campina Grande.

Walker, B., Holling, C. S., Carpenter, S. R., \& Kinzig, A. (2004). Resilience, adaptability and transformability in social-ecological systems. Ecology and Society, 9(2), 1-9.

Ward, S., Dornelles, F., Giacomo, M. H., \& Memon, F. A. (2019). Incentivising and charging for rainwater harvesting: three international perspectives. In F. A. Memon \& S. Ward (Eds.), Alternative Water Supply Systems (pp. 153-168). London: IWA Publishing.

Wilson, S., Pearson, L. J., Kashima, Y., Lusher, D., \& Pearson, C. (2013). Separating adaptive maintenance (resilience) and transformative capacity of social-ecological systems. Ecology and Society, 18(1), 1-12.

Wong, T. H. F., \& Brown, R. R. (2009). The water sensitive city: principles for practice. Water Science and Technology, 60(3), 673-682.

World Resources Institute - WRI. (2019). Water Risk Atlas. Recuperado em 16 de agosto de 2021, de https://www.wri.org/applications/aqueduct/water-risk-

atlas/\#/?advanced=false\&basemap=hydro\&indicator=w_awr_def_tot_cat\&lat $=-7.419121598981301 \& \operatorname{lng}=-$ $38.80810506641865 \&$ mapMode $=$ view $\&$ month $=1 \&$ opacity $=0.5 \&$ ponderation $=$ DEF \&predefined $=$ false $\&$ proj ection=absolute \&scenario=optimistic\&scope=baseline\&timeScale=annual\&year=baseline\&zoom=8

Zhang, X., \& Li, H. (2018). Urban resilience and urban sustainability: what we know and what do not know? Cities, 72, 141-148.

\section{Contribuições dos autores:}

Maycon Breno Macena da Silva: concepção do trabalho, escrita geral, levantamento bibliográfico, coleta de dados, análise e discussão dos resultados.

Daniela de Freitas Lima: escrita geral, levantamento bibliográfico, revisão geral do artigo, análise e discussão dos resultados.

Márcia Maria Rios Ribeiro: revisão geral do artigo, análise e discussão dos resultados. 